\title{
Utilisation of critical care services for surgical patients in a model three hospital
}

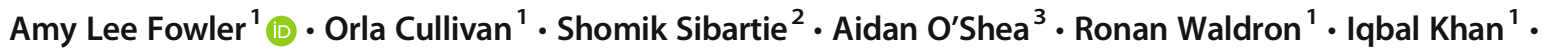 \\ Waqar Khan ${ }^{1} \cdot$ Kevin M. Barry ${ }^{1}$
}

Received: 10 October 2018 / Accepted: 30 January 2019 / Published online: 9 February 2019

(C) Royal Academy of Medicine in Ireland 2019

\begin{abstract}
Background The demand for intensive care unit (ICU) beds in the surgical population has increased in recent years. This is due to increased complexity of operative interventions, development of critical care services and improved availability of technologies. The number of beds in ICUs nationwide remains limited. In model three hospitals, this is further impacted by a lack of high dependency unit (HDU) facilities and difficulty with transfer of patients to tertiary centres.

Aim To assess utilisation of ICU resources amongst general surgical patients admitted for elective and emergency procedures to Mayo University Hospital.

Methods A prospective study was conducted between 31/10/2016 and 01/11/2017 on general surgical patients admitted to the intensive care unit. The ICU register and ICU database were used to collect data regarding patient demographics, admission by specialty, ICU length of stay, interventions performed, level of care, infection status and antimicrobial usage.

Results Eight hundred seventy-three patients were admitted to the ICU. One hundred thirty-four $(15.35 \%)$ were surgical admissions, of which 55 were elective and 79 were emergency. The most common cause for emergency admission to ICU was emergency laparotomy. Mean ICU length of stay (LOS) for surgical patients was 3.6 days. Three (2.2\%) surgical patients were transferred to model four hospitals.

Conclusions This study demonstrates the need to protect sufficient numbers of ICU beds for delivery of emergency surgical care. It highlights the potential utility of an HDU in this setting. The introduction of such a facility would impact cost savings and increase access for those requiring definitive ICU level care.
\end{abstract}

Keywords Critical care $\cdot$ Intensive care $\cdot$ Model three hospital $\cdot$ Resource utilisation $\cdot$ Surgery

\section{Introduction}

The role of critical care in the delivery of healthcare is evolving and the demand for intensive care unit (ICU) beds in the surgical population is rising internationally [1]. Many factors have been cited for this including improved practice of critical

Amy Lee Fowler

amyleefowler@rcsi.com

1 Department of General Surgery, Mayo University Hospital, Castlebar, Co. Mayo, Ireland

2 Department of Microbiology, Mayo University Hospital, Castlebar, Co. Mayo, Ireland

3 Department of Anaesthesia, Mayo University Hospital, Castlebar, Co. Mayo, Ireland care medicine, increased complexity of surgical intervention and the growth of critical care techniques and technologies [2]. Furthermore, variations in the number of ICU beds, staffing shortages and delayed discharge from ICU due to overall hospital bed shortages compound the issue [2]. In 2012, an estimated 289 critical care beds were in existence in Ireland ( 6.5 critical care beds per 100,000 population), placing Ireland amongst countries with the lowest critical care capacity worldwide [3].

In 2013, the Health Services Executive (HSE) issued a report to redistribute delivery of acute medicine in Ireland, followed by a similar outline for delivery of acute surgical services. These reports introduced a number of hospital 'models' $[4,5]$. The model three hospital consists of undifferentiated acute medical and surgical care, an acute medical assessment unit, an emergency department and a category 1 or 2 ICU [5]. In this setting, the shortage of available ICU beds 
is impacted by a lack of high dependency facilities and difficulty with transfer of patients to tertiary centres. Critical care beds are utilised for a wide case-mix of general surgical cases including trauma, emergency general surgery and postoperatively for major elective surgery [6].

Utilisation of ICU beds is further impacted by the rise in multidrug-resistant organisms in this setting such as methicillin-resistant Staphylococcus aureus (MRSA), vancomycin-resistant Enterococci and extended spectrum beta lactamases (ESBL) which prolong hospital length of stay, promote use of reserve antibiotics and further consume ICU resources [7].

Undeniably, the number of ICU beds available nationwide is limited. It is fundamental to understand our own use of ICU resources as a general surgical service, the level of critical care intervention received and the case-mix of general surgery patients using ICU services in our hospital. The aim of our study is to assess utilisation of ICU resources amongst general surgical patients admitted for elective and emergency procedures to Mayo University Hospital.

\section{Materials and methods}

A prospective study was conducted between 31/10/2016 and $01 / 11 / 2017$ on general surgical patients admitted to the intensive care unit at Mayo University Hospital (MUH). Mayo University Hospital is a teaching hospital with a total inpatient bed compliment of 332 beds, serving a catchment population of 130,638 [8] including 8 shared critical care beds ( $2.4 \%$ of overall bed capacity). The nursing ratio is $1: 1$ for ICU patients. Our ICU is fully equipped to provide levels 1, 2 and 3 care according to National Standards (see Table 1).

The ICU register, ICU database, Microbiology database and Electronic Discharge Summary were used to collect data regarding patient demographics, admission by specialty, ICU length of stay, overall length of stay, interventions performed, level of care, infection status and antimicrobial usage in this time period. In MUH ICU, microbiology ward rounds are conducted on a daily basis (Monday-Friday) with Microbiology on call service available. All antimicrobial usage in ICU is supervised by the 'Consultant Microbiologist'.

Data was entered and analysed using Microsoft Excel (2011). Ethics approval for this study was sought and obtained from Mayo University Hospital Ethics Committee.

\section{Results}

\section{Case-mix}

During the study period, there were a total of 873 admissions to MUH ICU, including 134 surgical admissions (15.7\%). This includes 13 repeat admissions in 6 patients. $41.0 \%(n=$ $55)$ were elective and 59\% $(n=79)$ were emergency surgical admissions. A total of 430 ICU bed days were used by surgical patients, with a range in length of stay (LOS) of 0-41 days, a mean LOS of 3.6 days and a median LOS of 2 days. The mean overall length of hospital stay for surgical patients admitted to ICU was 20 days, with a median of 6.5 days. Five deaths occurred in ICU and 3 surgical patients required transfer to a tertiary centre for head trauma, requiring Level $3 \mathrm{~S}$ neurosurgical care.

The most common elective postoperative admission to ICU was following anterior resection, $(38.2 \%, n=21)$ and for emergency admission, was emergency laparotomy (24\%, $n=19$ ) (see Fig. 1).

\section{Level of ICU care}

Throughout their ICU stay, $22.4 \%(n=30)$ of surgical patients received active treatment (including non-invasive ventilation, intubation and ventilation, tracheostomy, haemofiltration, nutritional support and pacing). The majority $(77.6 \%, n=104)$ received invasive monitoring alone via central venous or
Table 1 Levels of critical care, National Standards for Adult Critical Care Services (2011) [9]

\begin{tabular}{|c|c|c|}
\hline Level & Scope of intervention & Environment required \\
\hline $0 / 1$ & $\begin{array}{l}\text { No intervention needed } \\
\text { Simple non-invasive ventilation }\end{array}$ & $\begin{array}{l}\text { Resourced Observational Ward } \\
\text { Coronary/Acute Care Unit } \\
\text { Preoperative Assessment Unit }\end{array}$ \\
\hline 2 & $\begin{array}{l}\text { Increased frequency of monitoring } \\
\text { Complex non-invasive ventilation } \\
\text { Single organ failure }\end{array}$ & High Dependency Unit \\
\hline 3 & Management of two organ failures (or greater) & Intensive Care Unit \\
\hline $3 \mathrm{~S}$ & $\begin{array}{l}\text { Extra Corporeal Life Support } \\
\text { Neurological care } \\
\text { Cardiothoracic care } \\
\text { Post transplant care }\end{array}$ & Regional/National Specialty Unit \\
\hline
\end{tabular}


Fig. 1 Elective and emergency surgical procedures requiring admission to ICU

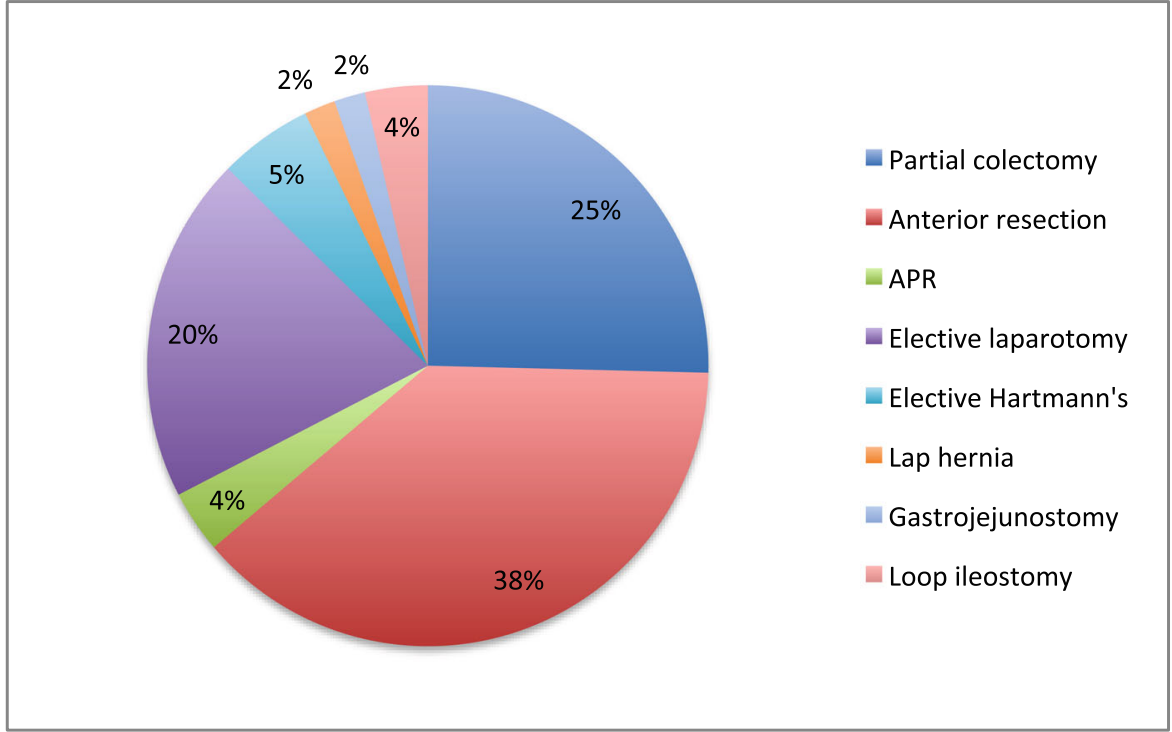

臭

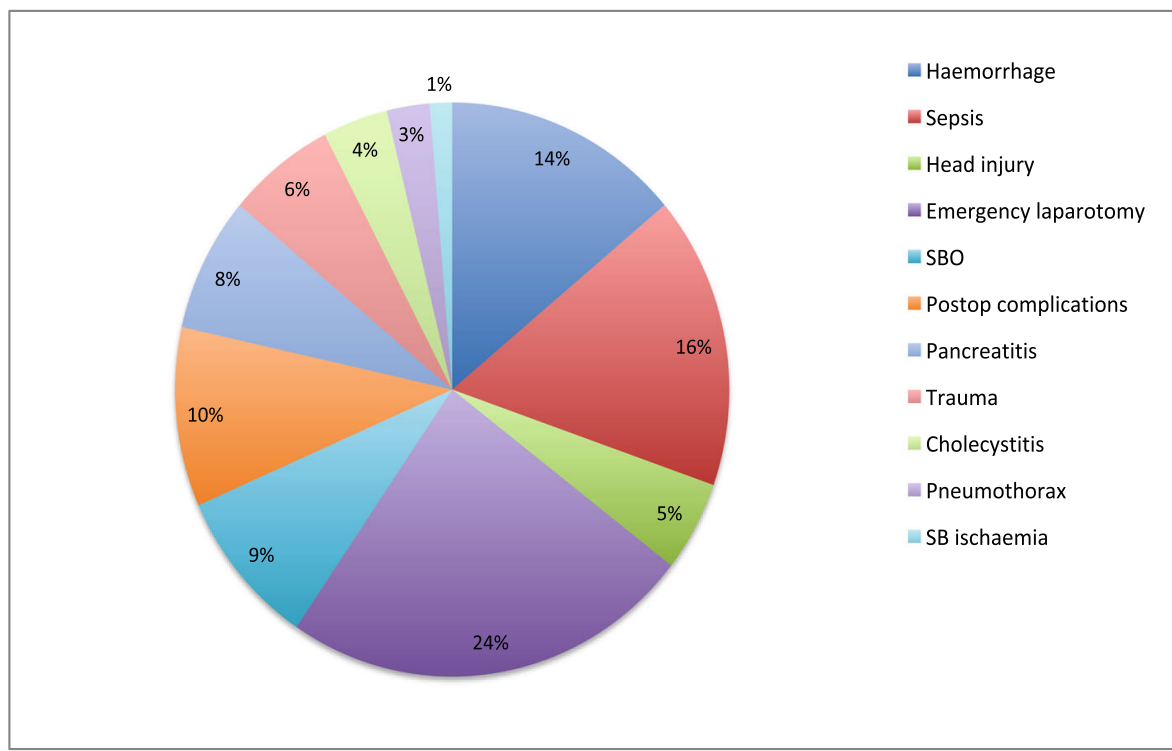

arterial line. Levels of care as defined by the National Standards for Adult Critical Care Services (2011) are described in Table 1 . The majority of elective $(81.8 \%, n=45)$ and emergency patients $(51.9 \%, n=41)$ required level 2 care due to need for increased frequency of monitoring (Fig. 2).

\section{Healthcare-associated infection status}

Regarding infection status during admission, $4 \%(n=5)$ of surgical patients were MRSA positive, $3 \%(n=4)$ of patients were ESBL positive and $3 \%(n=4)$ of patients were VRE positive. There was considerable use of reserve antimicrobials amongst this cohort, as seen in Table 2. Antifungals were used in $20 \%(n=11)$ of patients.

\section{Discussion}

Acute hospital beds including those in critical care facilities are in high demand and short supply in Ireland. The numbers of acute hospital beds are amongst the lowest in the world, at 3.0 per 1000 in 2015 according to OECD data [10]. Mayo University Hospital has a total acute bed capacity of 332 beds, including 8 shared critical care beds $(2.4 \%$ of overall bed capacity), serving a population of 130,638 giving $6.1 \mathrm{ICU}$ beds $/ 100,000$ population [8]. This is amongst the lowest ICU bed capacity worldwide [2, 10]. In 2017, the Critical Care Programme was launched to improve critical care performance, facilitate critical care bed planning and strengthen capability of critical care units [11]. In order to facilitate this, 
Fig. 2 Level of care required for emergency and elective patients

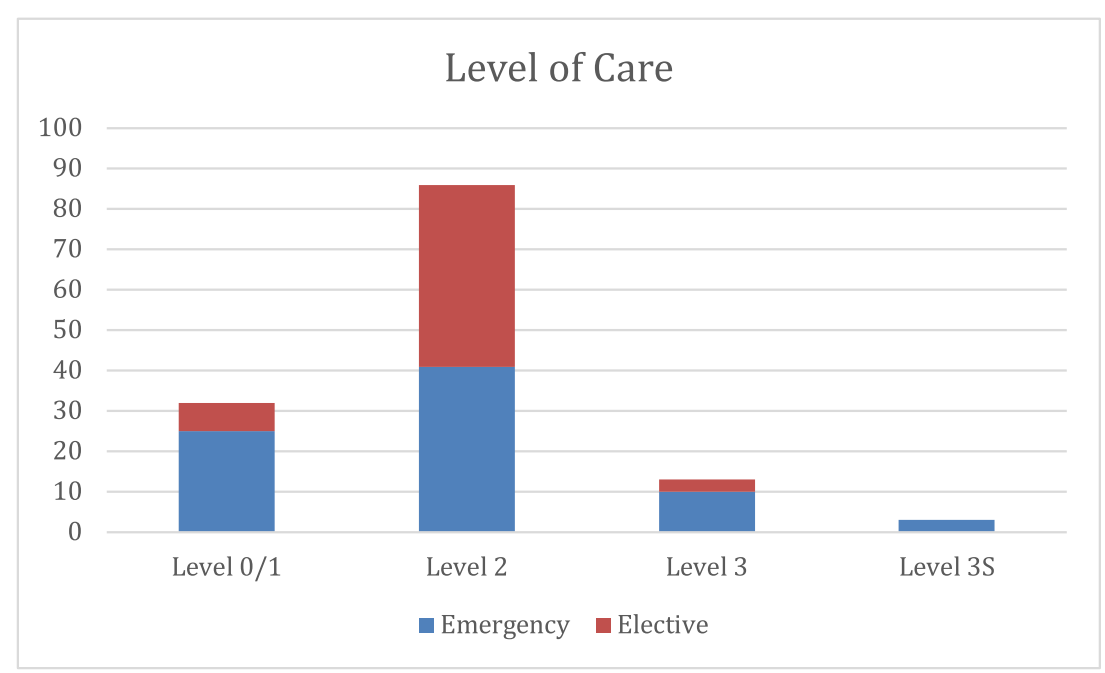

the National Office for Clinical Audit (NOCA) have launched an Irish National ICU audit to assess the activity, quality and complexity of care delivered in ten intensive care units across the country [12]. With this information, it is hoped that implementation of a 'Model of Care for Adult Critical Care' will be developed to ensure improved and efficient delivery of ICU resources [11].

Across many countries, studies have proposed that critical care provision is unlikely to meet estimated future demands $[3,13]$. Moreover, refusal or delay in admission to ICU due to limited bed capacity is associated with higher hospital mortality rates [14]. It remains critically important to understand the use of resources on an individual, local and national level in order to better utilise facilities, plan for future needs and distribute resources more efficiently [2].

General surgery in MUH comprises both emergency and elective treatment and covers a distinct case-mix and a geographically diverse population. Despite being a model 3 hospital, our critical care facility facilitated only three patient transfers to tertiary or quaternary centres for

Table 2 Antimicrobial usage amongst surgical patients in the ICU

\begin{tabular}{ll}
\hline Reserve antimicrobial type & Usage \\
\hline Tazocin & $29 \%(n=37)$ \\
Gentamycin & $20 \%(n=26)$ \\
Vancomycin & $11.8 \%(n=15)$ \\
Meropenem & $7.8 \%(n=10)$ \\
Linezolid & $5.5 \%(n=7)$ \\
Andilufungan & $4.7 \%(n=6)$ \\
Fluconazole & $4 \%(n=5)$ \\
Aztreonam & $0.8 \%(n=1)$ \\
Colistin & $0.8 \%(n=1)$ \\
Tigecycline & $0.8 \%(n=1)$ \\
\hline
\end{tabular}

intervention. Our results demonstrate that $64.2 \%(n=86)$ of patients were suitable for level 2 or high dependency standard care. $23.9 \%(n=32)$ required no intervention or active monitoring. Our results show $41 \%$ of cases admitted to the ICU were following elective procedures. Elective postoperative admissions to critical care facilities are as a result of the theoretical risk of postoperative complications and short-term postoperative mortality [15]. A recent study by Kahan et al. analysed 44,814 patients across 27 countries undergoing elective surgery and demonstrated no survival benefit associated with admission to critical care facilities post elective surgery [15]. Lack of availability of postoperative ICU beds has the potential to delay elective surgery. Frequently, patients admitted to ICU after elective surgery require management of analgesia, fluid balance, maintenance of nutrition and occasionally treatment for mild cardiorespiratory issues [16].

Emergency general surgery patients require significantly more ICU resources, have longer ICU length of stay and require higher rates of continuous renal replacement therapy, ventilator usage and other ICU interventions than nonemergency patients [6]. Our study demonstrated $59 \%$ of emergency surgery patients requiring ICU, the majority of which were general surgery patients.

We also analysed the impact of multidrug-resistant organisms and use of antibiotics in our ICU surgical patient population. Intensive care units are suitable environments for the development of multidrug-resistant organisms (MDRO) [17]. Seven percent of patients in our critical care facility were colonised or infected with multidrugresistant organisms. Furthermore, we also demonstrated a high utilisation of 'reserve' and multiple antimicrobial agents, which represent a risk factor for development of MDROs [18]. Prolonged length of stay prior to ICU admission, use of more than two types of antibiotics, 
duration of antimicrobial usage and recent use of broad spectrum antibiotics have all been mentioned as risk factors for MDRO colonisation [18]. This necessitates use of isolation beds and also represents a risk to other vulnerable, sick patients. Inappropriate patient selection puts patients at unnecessary risk of iatrogenic complications of ICU admission.

Use of ICU facilities comes at significant cost at a time when stringent resource utilisation for an increased patient population is needed [13]. The nature of ICU bed usage is changing with more beds being occupied by sicker, elderly patients and for end of life care $[1,6]$. Whilst an argument exists for the need for increased bed numbers, excess ICU beds in the USA have been associated with higher financial costs, increased ICU-acquired infections, immobility and 'overaggressive' end of life care [1]. Furthermore, higher numbers of ICU beds come at an increased cost and need for increased staffing rates, putting further strain on an already financially strapped system [1]. This raises the possibility of 'tiered' critical care facilities and the need for appropriate patient selection for the level of care required [19]. These findings were mimicked in an audit carried out by the Health Services Executive, who suggested that a large proportion of critical care facilities deliver only level 1/0 care, with few patients requiring higher levels of intervention. This necessitates reconfiguration of the delivery of ICU resources, so that critical care facilities would be delivered as a 'network', with greater numbers of beds in national specialty units or 'hub' hospitals and smaller services with step down facilities in 'spoke' hospitals [19].

Limitations of this study include the lack of assessment of severity of illness in this patient cohort, which would be useful to assess appropriate use of ICU facilities. Length of stay and number of transfers were low in our study, which may reflect the high level of care delivered in our facility. This would be further supported by assessment of severity of illness and may be reflective of the 'snapshot' of data used. A follow-up study is planned to include severity of illness data.

\section{Conclusions}

In model 3 hospitals, critical care facilities are limited and few high dependency units exist. Improved understanding of ICU bed usage helps to facilitate need for and planning of critical care resources. Introduction of HDU facilities would potentially result in cost savings and result in improved access for those definitively requiring ICU level care. This study highlights the importance of effective critical care facility use, the need for audit to highlight pitfalls in ICU resource utilisation and barriers to high quality ICU care.

\section{Compliance with ethical standards}

Ethics approval for this study was sought and obtained from Mayo University Hospital Ethics Committee.

Publisher's note Springer Nature remains neutral with regard to jurisdictional claims in published maps and institutional affiliations.

\section{References}

1. Ward NS, Chong DH (2015) Critical care beds and resource utilization: current trends and controversies. Semin Respir Crit Care Med 36(6):914-920. https://doi.org/10.1055/s-00351564876

2. Murthy S, Wunsch H (2012) Clinical review: international comparisons in critical care - lessons learned. Crit Care (London, England) 16(2):218. https://doi.org/10.1186/cc11140

3. Rhodes A, Ferdinande P, Flaatten H, Guidet B, Metnitz PG, Moreno RP (2012) The variability of critical care bed numbers in Europe. Intensive Care Med 38(10):1647-1653. https://doi.org/10. 1007/s00134-012-2627-8

4. Keane F MK (2013) Model of care for acute surgery. http://www. rcsi.ie/files/surgery/docs/20131030121710_RCSI_Model_of Care_for_Acute_S.pdf. Accessed 11 Nov $201 \overline{7}$

5. O'Neill S CG, Carroll J, Geary U, O'Reilly O, O'Connor M, Kearns B, Croke E, Whelan E, Scanlon S, Keown, A, Flynn M, Rafferty P (2010) Report of the National Acute Medicine Programme. https://www.hse.ie/eng/services/publications/clinicalstrategy-and-programmes/report-of-the-national-acute-medicineprogramme.pdf. Accessed 11 Nov 2017

6. Lissauer ME, Galvagno SM Jr, Rock P, Narayan M, Shah P, Spencer H, Hong C, Diaz JJ (2014) Increased ICU resource needs for an academic emergency general surgery service*. Crit Care Med 42(4):910-917. https://doi.org/10.1097/ccm. 000000000000099

7. Martin-Loeches I, Diaz E, Valles J (2014) Risks for multidrugresistant pathogens in the ICU. Curr Opin Crit Care 20(5):516524. https://doi.org/10.1097/mcc.0000000000000124

8. Office CS (2011) Census Results. http://census.cso.ie/areaprofiles/ PDF/CTY/mayo.pdf. Accessed 11 Nov 2017

9. Ireland JFoICMo (2011) National Standards for Adult Critical Care Services. https://www.anaesthesia.ie/attachments/article/57/ JFICMI_Minimum_Standards\%20Rev-01.pdf. Accessed 11 Nov $201 \overline{7}$

10. OECD (2015) Hospital beds. https://data.oecd.org/healtheqt/ hospital-beds.htm. Accessed 11 Nov 2017

11. Critical Care (2017) https://www.hse.ie/eng/about/who/cspd/ncps/ critical-care/. Accessed 11 Nov 2017

12. Audit NOoC National ICU Audit. https://www.noca.ie/audits. Accessed 11 Nov 2017

13. Sinuff T, Kahnamoui K, Cook DJ, Luce JM, Levy MM (2004) Rationing critical care beds: a systematic review. Crit Care Med 32(7):1588-1597

14. Prin M, Wunsch H (2012) International comparisons of intensive care: informing outcomes and improving standards. Curr Opin Crit Care 18(6):700-706. https://doi.org/10.1097/MCC. 0b013e32835914d5

15. Kahan BC, Koulenti D, Arvaniti K, Beavis V, Campbell D, Chan M, Moreno R, Pearse RM (2017) Critical care admission following elective surgery was not associated with survival benefit: prospective analysis of data from 27 countries. Intensive Care Med 43(7):971-979. https://doi.org/10.1007/ s00134-016-4633-8 
16. Pearse RM, Holt PJ, Grocott MP (2011) Managing perioperative risk in patients undergoing elective non-cardiac surgery. BMJ 343: d5759. https://doi.org/10.1136/bmj.d5759

17. Szabo M, Kanasz N, Darvas K, Gal J (2017) Identification of risk factors of multiresistant infections on two intensive care units. Orv Hetil 158(32):1259-1268. https://doi.org/10.1556/650.2017.30820
18. Huang X, Li G, Yi L, Li M, Wang J (2015) The epidemiology of multidrug-resistant bacteria colonization and analysis of its risk factors in intensive care unit. Zhonghua wei zhong bing ji jiu yi xue 27(8):667-671. https://doi.org/10.3760/cma.j.issn.2095-4352. 2015.08.010

19. Executive HS (2009) Towards excellence in critical care 